\title{
Single atoms on a roll
}

one of
the main
experimental
and
computational
challenges is
to stabilize
the single
Pt atoms
after the low
temperature
oxidation of
co

Many indispensable commodity chemicals are synthesized using heterogeneous catalysis, which owes a lot to platinum group metals especially in the form of nanoparticles. When a catalyst features a precious metal, it is particularly important to ensure that each of these atoms is an efficient participant. This has motivated us to downsize metal nanoparticle catalysts to single atoms. Such catalyst engineering is not only driven by economic factors but may also enable reactivity not accessible to bulk species. For example, single metal atoms dispersed on solid substrates are remarkably active for $\mathrm{CO}$ oxidation and the water-gas shift reaction. Yet, despite the many reports of single atom catalysts, our understanding of the chemistry at an atomic level is limited. Indeed, there is often only scant evidence that the isolated metal atoms in certain systems stay isolated under catalytic conditions.

The surface of a heterogeneous catalyst can feature metal active sites in a many different chemical environments. This complexity makes it difficult to identify a sufficiently simple catalyst

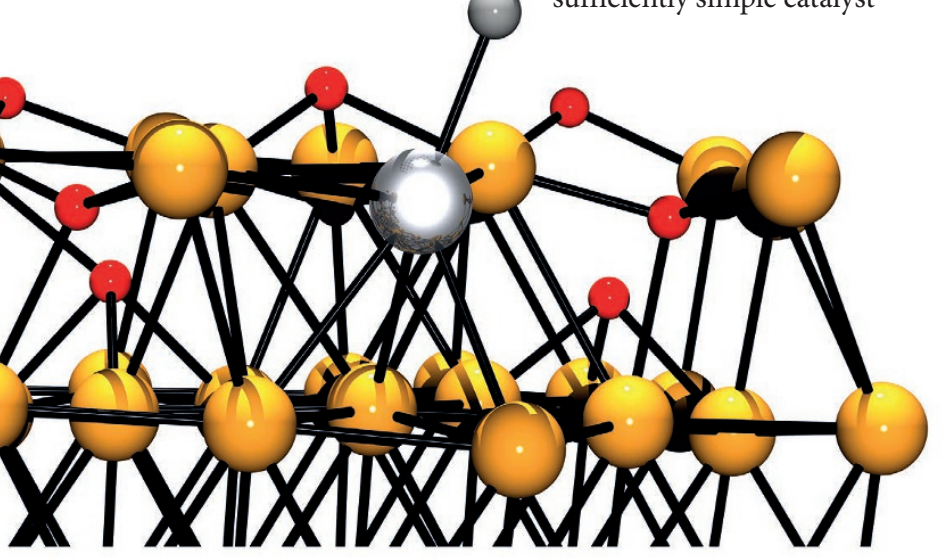

model that mimics the individual catalytic sites and allows us to uncover novel reaction mechanisms. Addressing this challenge, Jean-Sabin McEwen, Charles Sykes and colleagues describe in Nature Catalysis a well-defined material featuring $\mathrm{Pt}$ atoms on a monolayer $\mathrm{Cu}_{2} \mathrm{O}$ (111) support. Deposited from Pt evaporated with an electron beam, the nature of the Pt on the surface can be precisely controlled and can be anything from uniform extended surfaces to isolated atoms. With the new materials in hand, the team sought to study the catalytic activity for the oxidation of $\mathrm{CO}$ to $\mathrm{CO}_{2}$, a reaction that typically requires high temperatures - conditions under which many single atom catalysts are deactivated. Encouragingly, the team found that highly dispersed $\mathrm{Pt}$ atoms can remain isolated, with infrared spectra featuring a low energy $v_{\mathrm{CO}}$ band consistent with these sites binding a single $\mathrm{CO}$ molecule when exposed to the gas. Spectra of samples with higher $\mathrm{Pt}$ coverages feature a higher energy $v_{\mathrm{CO}}$ band on account of the non-trivial dipole-dipole interactions between nearby CO molecules.

Having established the robustness of their system, McEwen, Sykes and co-workers used a suite of analytical techniques to probe the conversion of $\mathrm{CO}$ into $\mathrm{CO}_{2}$. Temperature programmed desorption enabled them to show that single Pt atoms are indeed active in the oxidation reaction. Moreover, the team found that the single atoms are more resilient towards $\mathrm{CO}$ poisoning than are Pt clusters and nanoparticles. This positive result was followed by isotopic labelling and scanning tunnelling microscopy experiments, which enable the team to visualize the consumption of an $\mathrm{O}$ atom in the $\mathrm{Cu}_{2} \mathrm{O}$ lattice in close vicinity to a $\mathrm{Pt}$ site in the oxidation process. X-ray photoelectron spectra indicated that these active single Pt atoms are charge-neutral under the catalytic conditions. This may come as a surprise to inorganic chemists, who only rarely encounter $\mathrm{Pt}(0)$ carbonyls species that are typically very labile. The team concluded that their system operates by a Mars-van Krevelen mechanism, whereby the $\mathrm{C}$ atom of a Pt-CO group is directly attacked by a lattice $\mathrm{O}$ atom; no high-valent intermediates such as $\mathrm{Pt}-\mathrm{O}$ species are involved.

The present work is exciting in that $\mathrm{CO}$ oxidation proceeds even under mild conditions - the team's operating temperature of $345 \mathrm{~K}$ is much lower than the $425 \mathrm{~K}$ target for mitigating motor vehicle emissions. However, "one of the main experimental and computational challenges is to stabilize the single Pt atoms after the low-temperature oxidation of CO”, says McEwen. The catalysts lose activity over time because the $\mathrm{Pt}$ atoms move into the layers of the $\mathrm{Cu}_{2} \mathrm{O}$ support, where they remain highly dispersed but unable to bind $\mathrm{CO}$. The team is now studying a host of oxide-metal interactions to identify a stable system capable of mediating the entire catalytic cycle of this important reaction.

Yaoqing Zhang, Associate Editor, Nature Communications

ORIGINAL ARTICLE Therrien, A. J. et al. An atomic-scale view of single-site Pt catalysis for low-temperature CO oxidation. Nat. Catal. 1, 192-198 (2018) 